\title{
MEDICINA HUMANA ESPACIAL: PERFORMANCE FISIOLÓGICO Y CONTRAMEDIDAS PARA MEJORAR LA SALUD DEL ASTRONAUTA
}

\author{
HUMAN SPACE MEDICINE: PHYSIOLOGICAL PERFORMANCE AND COUNTERMEASURES TO IMPROVE \\ THE ASTRONAUT'S HEALTH \\ Milton V. Rivera ${ }^{1, a}$, José Cornejo ${ }^{1, b}$, Karen Huallpayunca ${ }^{1, c}$, Aurora B. Diaz ${ }^{1, d}$, Zhamanda N. Ortiz-Benique ${ }^{1, e}$, \\ Andrés D. Reina ${ }^{1, f}$, Gustavo Jamanca Lino ${ }^{1,9}$, Victor Ticllacuri ${ }^{1, h}$
}

\begin{abstract}
RESUMEN
Se presenta este artículo de revisión con base en la evidencia científica actual sobre medicina espacial enfocada en fisiología humana y sus contramedidas. Por lo cual se realizó una búsqueda bibliográfica no sistemática de artículos científicos y libros de investigación en inglés-español de los últimos 7 años, que detallan su aplicación en seres humanos, modelos murinos y experimentos in vitro. Se tomaron en cuenta las condiciones del ambiente espacial como microgravedad y radiación que producen considerables cambios fisiológicos en el sistema cardiovascular (redistribución de líquidos, remodelación cardiovascular, arritmias); nervioso (sensitivomotores, neurosensoriales, neurovestibulares); respiratorio (cambios de volúmenes y capacidades); renal (litiasis); musculoesquelético (atrofia muscular, osteoporosis); hematológico (anemia); inmunológico (desregulación inmune) y digestivo (alteración de la microbiota intestinal). Además, existen procesos biológicos, moleculares y genéticos aún por explorar, para conocer y mitigar los mecanismos inciertos desencadenados en ambientes extremos y peligrosos. Por lo tanto, es una prioridad desarrollar e implementar contramedidas para reducir los efectos nocivos en la salud, con el objetivo de garantizar la adaptación, seguridad y performance del astronauta durante futuros viajes espaciales.
\end{abstract}

Palabras clave: Medicina espacial; Fisiología humana; Contramedidas; Microgravedad; Radiación; Salud; Adaptación; Seguridad; Performance; Astronauta (fuente: DeCS BIREME).

\begin{abstract}
This Review Article is presented based on current scientific evidence on space medicine focused on human physiology and its countermeasures. Therefore, a non-systematic bibliographic search of scientific articles and research books in English-Spanish of the last 7 years was carried out, detailing their application in humans, murine models and in vitro experiments. The conditions of the space environment such as microgravity and radiation that produce considerable physiological changes in the cardiovascular system (redistribution of fluids, cardiovascular remodeling, arrhythmias) were taken into account; nervous (sensorimotor, neurosensory, neurovestibular); respiratory (volume and capacity changes); renal (lithiasis); musculoskeletal (muscular atrophy, osteoporosis); hematological (anemia); immunological (immune dysregulation) and digestive (intestinal microbiota disorder). In addition, there are biological, molecular and genetic processes still to be explored, in order to know and mitigate the uncertain mechanisms triggered in extreme and dangerous environments. Therefore, it is a priority to develop and implement countermeasures to reduce the harmful effects on health, with the aim of guaranteeing the astronaut's adaptation, safety and performance during future space flights.
\end{abstract}

Key words: Space medicine; Human physiology; Countermeasures; Microgravity; Radiation; Health; Adaptation; Safety; Performance; Astronaut (source: MeSH NLM).

\footnotetext{
1 Space Medicine and Biomechatronics Research Group - The Mars Society Peru, Lima-Perú.

a BSc in Nursing \& Medical Doctor Candidate (Perú) - Human Physiology Specialist.

${ }^{b}$ MBA \& MSc, Bio Mechatronics Engineer (Perú) - General Coordinator.

MSc(C) Biochemistry \& Occupational Medicine and Environment, Medical Doctor (Perú) - Space Human Medicine Science Manager.

Medical Doctor Candidate (Perú) - Space Agricultural Medicine Science Manager.

e Medical Doctor Candidate (Perú) - Human Medicine Project Member.

${ }^{f}$ Aeronautical Engineer, Technician in Non Destruction Testing (Colombia) - Space Veterinary Medicine Science Manager.

g MSc Geometallurgy Candidate (Perú) - Astrophysics Specialist.

h Biomedical Engineer Candidate (Perú) - Engineering Project Member.
}

Citar como: Milton V. Rivera, José Cornejo, Karen Huallpayunca, Aurora B. Diaz, Zhamanda N. Ortiz-Benique, Andrés D. Reina, Gustavo Jamanca Lino, Victor Ticllacuri. Medicina humana espacial: performance fisiológico y contramedidas para mejorar la salud del astronauta. Rev. Fac. Med. Hum. Abril 2020; 20(2):303-314. DOI 10.25176/RFMH.v20i2.2920 


\section{INTRODUCCIÓN}

Desde tiempos prehistóricos, el ser humano se ha interesado por observar las estrellas en el oscuro cielo ${ }^{(1)}$. Desde entonces, el soñar, explorar, descubrir y conocer son los primeros pasos realizados por el hombre ${ }^{(2)}$. La ciencia, paralelamente con el avance tecnológico, ha logrado revelar muchos de los más profundos secretos del $\operatorname{cosmos}^{(3)}$. Hemos podido viajar fuera de nuestra atmosfera terrestre y quizá, en el futuro, podamos internarnos en su inmensidad convirtiéndonos en una nueva especie colonizadora espacial. La existencia de vida en condiciones extremas sigue siendo un gran desafío desde el punto de vista médico-biológicotecnológico.

En ese sentido, "La Medicina Espacial" es una rama de la medicina aeroespacial que estudia la adaptación del ser humano en relación a su respuesta al ambiente de microgravedad y a la alta radiación en la órbita terrestre existente más allá de los Campos de Van Allen ${ }^{(1)}$. A raíz de los primeros intentos de llevar al hombre al espacio, los proyectos iniciales para alcanzar dicho objetivo requirieron resolver varios problemas en soporte vital, seguridad e higiene. Desde entonces es cuando la medicina espacial se consolida como ciencia dedicada al estudio, control y acondicionamiento del cuerpo fuera de nuestro planeta ${ }^{(4)}$. Además, la medicina espacial define los riesgos para la salud humana durante los vuelos espaciales de corta y larga duración, asimismo propone las medidas terapéuticas para revertir desórdenes fisiológicos, bioquímicos y genéticos que aún no están descritos por completo ${ }^{(5,6)}$.También, diseñará en el futuro nuevos protocolos de prevención, diagnóstico y tratamiento en misiones de emergencia; la preservación de la salud humana es una de las metas principales para la comunidad científica espacial con el objetivo de llevar a cabo viajes interplanetarios para fines de exploración, terraformación y colonización. En la actualidad, muchos programas académicos de ciencias espaciales, vienen incrementándose en todo el mundo siendo un dominio de colaboración y cooperación entre naciones en las diferentes ramas de la carrera espacial ${ }^{(7)}$.

Por ello, el objetivo del presente artículo es ampliar los conocimientos en medicina espacial respecto a los efectos fisiológicos y sus contramedidas; empleando las siguientes bases de datos: Google Scholar, PubMed-NCBI, SpringerLink, Scopus-Elsevier, y SciELO. Los resultados se sintetizan en 3 áreas temáticas: Clima espacial, Síndrome de adaptación espacial y Cambios fisiológicos (cardiovascular, nervioso y vestibular, musculo-esqueletico, respiratorio, renal, hematopoyético, inmunológico, gastrointestinal; y sus respectivas contramedidas). Los filtros fueron obtenidos de los últimos 7 años, los idiomas consultados fueron inglés y español, además se consideró estudios realizados en humanos, modelos murinos y experimentos in vitro en condiciones espaciales de microgravedad real y simulada. Seguidamente, se utilizó información en el servidor de informes técnicos de la NASA (Technical Reports Server).

URL: https://www.sti.nasa.gov/ debido a su política de trasparencia de publicar información de mucha importancia.

Se encontraron 103 bibliografías en las bases de datos consultadas, de los cuales 10 fueron excluidos por no contar con textos completos y además, no fueron desarrollados en humanos y murinos. Luego de la lectura de los mismos, 93 se incluyeron en esta revisión artículos como: originales de investigación, de revisión, express, informativos y reportes científicos referidos a las áreas temáticas ya establecidas en un inicio. De los mismos se incluyeron 9 artículos originales que cumplen con las variables correspondientes en la Tabla 1, donde se resumen los resultados de cada investigación.

\section{CLIMA ESPACIAL Y RIESGOS PARA LA SALUD}

La radiación espacial y la microgravedad son considerados dos de los factores principales que causan mayor influencia sobre la salud humana en entornos espaciales ${ }^{(8)}$. Los eventos mencionados conllevan un riesgo para los sistemas biológicos del Planeta Tierra, por lo tanto, sus funciones necesitan adaptarse para sobrevivir.

La dosis equivalente de radiación espacial es entre 200 a 400 mSv/año con efectos acumulativos sobre la salud ${ }^{(9)}$; en consecuencia, los riesgos que provoca en la salud son directamente proporcionales con el tiempo de viaje y la distancia recorrida por la nave con respecto a los campos de Van Allen. Estas fuentes de radiación proceden de los rayos cósmicos galácticos (GCR), partículas energéticas solares (SPE), más la radiación de los cinturones de Van Allen de la órbita terrestre ${ }^{(10)}$.

Los eventos de partículas solares (SPE), como las llamaradas o eyecciónes de la masa coronal (CME), emiten alta energía (rayos gamma o X), electrones energéticos, protones, partículas alfa y partículas pesadas $^{(11)}$. Adicionalmente, otra fuente de radiación es nuestra Galaxia "La Vía Láctea", que a través de los GCR emite partículas principalmente compuestas por $85 \%$ de hidrógeno, $14 \%$ de helio y un $1 \%$ de núcleos de alta energía (HZE), estos últimos son menos abundantes, pero en comparación con otras partículas, estas tienen mayor poder ionizante, de penetración y potencial de daño por radiación ${ }^{(9)}$. 
La microgravedad, es un ambiente donde el peso de los objetos se percibe reducido, y están bajo los efectos de gravedad mínima (valores cercanos a cero). Por ejemplo, la Estación Espacial Internacional (EEI) está sometida a un $90 \%$ de la gravedad terrestre promedio 1G (9.80665 $\left.\mathrm{m} / \mathrm{s}^{2}\right)^{(12)}$, sin embargo, al estar en permanente caída libre durante su trayectoria de vuelo orbital se experimenta un ambiente de microgravedad ${ }^{(13)}$, por ello las personas y objetos en la estación espacial parecen "flotar".

\section{SÍNDROMEDEADAPTACIÓN ALESPACIO}

Este síndrome, en sus siglas en inglés SAS (Space Adaptation Syndrome) denominado también "enfermedad del espacio", tiene una incidencia de hasta $60-80 \%$ en los miembros de la tripulación y consiste principalmente en la presencia de mareos a partir de la primera misión. Dura aproximadamente 72 h, tiempo en el que el cuerpo humano se acostumbra a su nuevo ambiente ${ }^{(14,15)}$.

Se observa que en exposiciones cortas a microgravedad, el sistema neurovestibular en su afán de adaptarse a este ambiente origina cambios fisiológicos; síntomas generales como: nauseas autocontrolables, vómitos, mareos, sudoración, palidez, malestar general. Otros síntomas son: vértigo, cefalea, aletargamiento, movimientos descoordinados y disminución en la concentración $^{(14,15)}$.

También se presentan síntomas digestivos como: incomodidad gástrica, anorexia, pérdida de apetito, disminución drástica de ruidos intestinales y vómitos en proyectil que culminan con el cuadro clínico ${ }^{(14,16)}$.

Las contramedidas para estas manifestaciones son: evitar las maniobras bruscas en el movimiento de la cabeza y el cuerpo, aplazar actividades físicas extremas en los primeros días de vuelo y el uso de la prometazina como tratamiento profiláctico que minimiza estos síntomas de manera efectiva ${ }^{(15,16,17)}$.

Se debe diferenciar el SAS de la Enfermedad de movimiento de entrada o "Entry Motion Space" (EMS) en inglés y Enfermedad de movimiento espacial o "Space Motion Sickness" (SMS) en inglés ${ }^{(15,17)}$.

La EMS, se presenta al momento de ingresar a la atmosfera terrestre (síndrome de readaptación a la tierra) sus síntomas son similares al SAS, sin embargo, ciertos investigadores creen que su presentación puede ser más severa en relación al mayor tiempo de vuelo, originando: malestar general, náuseas, cefalea, mareos, falta en el control de la postura cefálica, descoordinación, intolerancia ortostática, disminución de la fuerza muscular y debilidad física. La incidencia de los síntomas del EMS, es mayor en mujeres que en varones ${ }^{(17,18)}$.
La SMS ocurre durante el vuelo espacial y después de regresar al Planeta Tierra, los mecanismos de su desarrollo no son concluyentes, aún están en estudio. Sus manifestaciones son: ilusión de orientación, mareos, descoordinación, náuseas, vómitos, dificultad en la fijación en la mirada y localización de objetos ${ }^{(17,19)}$.

\section{CAMBIOS FISIOLÓGICOS}

\section{Sistema cardiovascular}

El ser humano se ha adaptado en la tierra bajo diferentes estados de aceleración, posición y condiciones de gravedad $1 \mathrm{G}^{(20)}$. Sin embargo, al entrar en un ambiente de microgravedad en las primeras fases de adaptación se altera la homeostasis y ocurre el desplazamiento o redistribución del líquido intravascular desde las extremidades inferiores a las extremidades superiores, tórax y región cefálica ${ }^{(21)}$; por tal razón, los astronautas experimentan síntomas de sensación de plenitud cefálica, congestión nasal, edema de cuerdas vocales y facial(22). De la misma forma, datos clínicos han demostrado que los cambios hemodinámicos son variables de acuerdo al tiempo de vuelo y posición del cuerpo; en las primeras 24 a 72 horas se genera un aumento del gasto cardiaco (GC), volumen sistólico (VS), dimensiones de las cavidades cardiacas y disminución de la presión venosa central (PVC). La frecuencia cardiaca (FC), presión arterial sistólica (PAS), presión arterial diastólica (PAD) y la presión arterial media (PAM) pueden disminuir o permanecer sin cambios en periodos prolongados de vuelo ${ }^{(23,24)}$.

En las adaptaciones en un tiempo mayor a 12 días ocurre la remodelación del miocardio, principalmente en el ventrículo izquierdo donde el espesor de la masa miocárdica disminuye en $11 \%{ }^{(23)}$. Durante las fases tardías, en una misión espacial con duración aproximada superior a 6 meses, el corazón disminuye su tamaño y volumen en 15 a 20\% (atrofia) ${ }^{(25)}$. La microgravedad induce la activación de este mecanismo a través de múltiples moléculas de señalización relacionadas con la autofagia y apoptosis de los miocardiocitos, como la proteína quinasa activada por AMP (AMPK), ERK1 / 2, LC3-II e histona desacetilasa 4 (HDAC4) ${ }^{(26)}$ con aumento de los marcadores séricos creatina quinasa (CK) y troponina (Tnc). Por lo tanto, el objetivo terapéutico para bloquear o inhibir el remodelamiento cardiaco es la proteína 1 (CKIP-1) debido a su capacidad de participar en las vías de señalización intracelular con interacción de diferentes proteínas ${ }^{(20)}$.

A nivel de los vasos sanguíneos existen datos clínicos de disfunción vascular inducida por radiación, dicho deterioro conduce a la enfermedad arterial oclusiva que podría ser un factor de riesgo determinante para el desarrollo de enfermedades cerebrovasculares (ECV) ${ }^{(27)}$. 
En la electrocardiografía (ECG) se suelen presentar alteraciones electrofisiológicas inducidas por cambios moleculares y celulares que originan canalopatías y posteriormente miocardiopatías ${ }^{(28)}$, así como la presencia de extrasístoles ventriculares aisladas, arritmias malignas, riesgo de inestabilidad en la re-polarización, contracción auricular prematura, incremento del complejo QRS y del intervalo PR e inclusive muerte cardiaca súbita ${ }^{(13,22,29)}$.

Sus contramedidas para evitar el desacondicionamiento cardiovascular requiere de participación activa del sujeto, con ejercicios físicos pasivos/activos, aeróbicos, trajes con presión negativa en la parte inferior del cuerpo de tipos (Penguin, Braslet, Chibis y Kentavr), plataformas de vibración ${ }^{(30,31,32)}$, protección contra la radiación, antioxidantes, nutracéuticos, medicamentos que ayuden a disminuir procesos inflamatorios y gravedad artificial a través de las centrifugaciones intravehiculares para la correcta redistribución de fluidos en el cuerpo ${ }^{(33)}$.

\section{Sistema nervioso y vestibular}

Los vuelos espaciales tienen efectos perjudiciales en la neurofisiología humana ${ }^{(34)}$, ambos sistemas se ven afectados por las ondas gravitacionales como las disfunciones en las áreas somatosensoriales corticales, sensitivomotores, neurooftálmicas y vías vestibulares ${ }^{(35)}$. La exposición a la microgravedad ocasiona cambios y déficits funcionales neurológicos como: debilidad neuromuscular, ataxia, desequilibrio, descoordinación, disfunción cognitiva, alteración en la motricidad, cambios psicológicos, problemas psiquiátricos, ilusión perceptual y aumento de la presión intracraneal (PIC). En definitiva, las condiciones de microgravedad ha demostrado alteraciones en estructura y función en el cerebro humano(35,36,37).

A nivel molecular, la microgravedad regula negativamente la expresión y la liberación de los neurotransmisores; por ejemplo el incremento de concentración del ácido glutámico (Glu) y reducción de 5-hidroxitriptamina (5-HT), dopamina (DA), ácido $\mathrm{Y}$-aminoácido butírico (GABA) y epinefrina (E), estos cambios están relacionados con la disminución del aprendizaje y la memoria(38). La mayor frecuencia de los casos se presenta en los vuelos espaciales de larga duración.

Los cambios de aceleración lineal y angular durante los vuelos espaciales repercuten notablemente en el sistema neurovestibular originando un desbalance de información proporcionada por parte del oído interno (canales semicirculares y otolitos), núcleos oculomotores (III y IV), cerebelo, médula espinal y sistema nervioso central (SNC) encargados de combinar información sensorial y motora. Tras estas alteraciones se producen: desorientación, desequilibrio postural, descoordinación de los movimientos, disfunción propioceptiva y alteración en el control de movimientos oculares $^{(36,39)}$. En vuelos espaciales de larga duración los otolitos se adaptan remodelando su estructura histológica, igualmente la sustancia gris en el cerebelo y en áreas sensorio motores del cerebro aumenta de espesor mejorando el control en los miembros inferiores $^{(40)}$.

La capacidad de la visión también se ve afectada, prueba de esto es que más de la mitad de astronautas retornan a la tierra de la EEl con alteraciones neurooculares; como edema en el disco óptico, distención de vaina del nervio óptico, aplanamiento del globo ocular, pérdida de reflejo vestíbulocular, aumento de la presión intraocular (PIO) ${ }^{(41,42)}$, oscilopsia, visión borrosa por el desplazamiento de la retina e incapacidad de mantener la imagen enfocada en la retina (hipermetropía) ${ }^{(43,42)}$.

Por tanto, la gravedad artificial a través de centrifugaciones intravehiculares y el diseño de dispositivos médicos de estabilización postural, conocidos como auxiliares vestibulares microgiróscopos y microacelerómetros (prótesis vestibular), son las opciones apropiadas para evitar alteraciones posturales, estabilizar el enfoque visual, trastornos somatosensoriales, sensitivomotores corticales, neurovestibulares, cambios neuroftálmicos y/o disminuir el desarrollo de conflictos sensoriales ${ }^{(31,39,44)}$.

\section{Sistema músculoesquelético}

A nivel muscular, en vuelos espaciales no se necesita de esfuerzo muscular como en la tierra, por ello los músculos desarrollan una adaptación negativa al ambiente de microgravedad, paralelamente la fuerza muscular disminuye de un 20 a $40 \%$ en las primeras semanas de su permanencia en el espacio. Los músculos antigravitatorios (músculos de la cadera, espalda, cuádriceps, gastrocnemio y los músculos del cuello) son mayormente afectados con atrofia a diferencia de los músculos flexores ${ }^{(13)}$.

Luego de 2 o 3 semanas el deterioro se hace más evidente(13), es decir, la "no carga" mecánica generada sobre los músculos ocasiona cambios en la composición de relación de las proteínas actina/ miosina e incluso la disminución de otras proteínas musculares como la titina y nebulina causados por desregulación negativa de muchos genes como por ejemplo (TGF1, Fzd9, Casq2, Kcnma1, Ppara, Myf6) ${ }^{(45,46)}$. En estos procesos se presentan las alteraciones en la transcripción de proteínas contráctiles y degradación a través de la vía ubicuitina-proteosoma de la apoptosis; evitando de esa manera su regeneración, crecimiento 
y desarrollo. También se han observado alteraciones en la homeostasis de calcio y en la respuesta al estrés oxidativo/inflamatorio por aumento de expresión de quimiocinas (CCL2, CCL7 y CCL12) en el músculo, este último se amplifica tanto por el desuso, como también por altas dosis de radiación generando pérdida de la masa en los principales músculos que se oponen a la gravedad $^{(46,47,48)}$.

A nivel óseo, al dejar de estar sometido a las fuerzas de gravedad se genera disminución del grado de tensión dentro del hueso y la baja iluminación presente en el espacio origina deficiencia de síntesis de la vitamina D3. A partir de estos cambios, se produce una acelerada descalcificación, ocasionando pérdida en la densidad ósea aproximadamente de 1 a $2 \%$ por mes específicamente en el cuello del fémur, vértebras, pelvis, tibia y calcáneo aumentando el riesgo de fracturas por fragilidad $^{(1,13)}$

En dicho proceso está involucrada la autofagia, con niveles acentuados de marcadores de genes (Atg5, LC3 y Atg16L), además el aumento de la expresión de la fosfolipasa PLC 2 , moléculas de señalización de proteínas quinasas activadas por mitógenos MAPK/ERK, P38 y activación de osteoclastos a través del receptor activador nuclear kappa beta (RANK) que promueven la reabsorción ósea ${ }^{(45,49,50)}$, con aumento de marcadores como el telopéptido c-terminal del colágeno tipo 1 (CXT) y telopéptido n-terminal del colágeno tipo 1 (NTX) en la orina. En concentraciones sanguíneas se observa incremento de la fosfatasa alcalina (FA), isoenzima ósea (bALP) y propéptido N-terminal de procolageno tipo I (PINP) (51). Es por esto que el bloqueo de la autofagia es una alternativa terapéutica para prevenir la pérdida ósea en el espacio, debido a que la supresión de la misma da como resultado una regulación negativa de la osteoclastogénesis ${ }^{(1,45,52)}$.

También, se evidencia que el disco intervertebral se ensancha por ganancia de agua debido a las condiciones de microgravedad, porque el peso diferencial que soportan los discos en el Planeta Tierra disminuye en el espacio; sin este esfuerzo mecánico de compresión discal se condiciona a un incremento en la estatura del astronauta entre 5 a $7 \mathrm{~cm}$ produciéndose así dolor en la zona lumbar ${ }^{(13)}$.

Para contrarrestar estos efectos mencionados, se recomienda utilizar la estimulación mecánica como los ejercicios de resistencia con cuerdas elásticas, dispositivos avanzados de ejercicio de resistencia (ARED), cicloergómetro con sistema de aislamiento de vibraciones (CEVIS), dispositivo de ejercicio de volante (FWED), sistema de estabilización y aislamiento de vibraciones $(T V I S)^{(32)}$, plataformas vibratorias y cinta de correr ${ }^{(53)}$.
En lo que respecta a nutrición (suplementos de calcio, proteínas, vitamina $D$, vitamina $K$ ) y la administración de bifosfonatos inhiben su reabsorción y mejoran la formación del hueso, ello también evita la degeneración neuromuscular ${ }^{(53,54,55)}$. La atrofia osteomuscular no ha podido ser evitada de manera completa, por lo cual aún es necesario realizar más investigaciones que permitan el desarrollo de nuevas tecnologías para controlar la disfunción muscular y ósea de manera más eficiente ${ }^{(55)}$.

\section{Sistema respiratorio}

La exposición a la microgravedad anula el efecto Slinky y la ventilación/perfusión (V/Q) es igual en todas las zonas de West, por lo tanto, el flujo sanguíneo es homogéneo en las zonas 1, 2 y 3 del pulmón. Lo contrario sucede en condiciones $1 \mathrm{G}$, es decir, la diferencia de presión entre los conductos arteriales, venosos y alveolares sobre las regiones mencionadas en los pulmones, es resultado directo de la gravedad del Planeta Tierra ${ }^{(56)}$.

En las misiones espaciales 1 y 2 de Spacelab se evidenció que la ingravidez en los primeros 17 días de vuelo causó un ligero incremento en la frecuencia respiratoria (FR) y los volúmenes pulmonares, reduciendo así la capacidad vital (CV) del 5 a $10 \%$, volumen tidal (VT) de $15 \%$ y capacidad vital forzada (CVF) de 3-5\%, debido al desplazamiento de vísceras abdominales y líquidos en sentido cefálico ${ }^{(4,24,51)}$. De la misma manera, la capacidad residual funcional (FRC) y el volumen residual (VR) disminuyeron en $15 \%$ y $18 \%$ respectivamente, debido al fenómeno de expansión alveolar uniforme. En vuelos de periodos prolongados, la FR, CV y la CVF recuperan sus valores normales ${ }^{(24)}$, es por ello que no existen cambios significativos incluso después de haber permanecido durante 6 meses al interior de la EE ${ }^{(56)}$.

El consumo de $\mathrm{O}_{2}$ y la producción de $\mathrm{CO}_{2}$ disminuyen en un periodo de 2 a 4 meses, probablemente por el trabajo muscular de la pared torácica y la carga gravitacional reducida en el espacio ${ }^{(51)}$. Asimismo, el intercambio de gases parece ser más eficiente en microgravedad real en comparación con la microgravedad simulada ${ }^{(56)}$.

Se ha visto que esta última influye en la composición del agente tensoactivo pulmonar y sus atributos, además promueve el cambio del equilibrio de líquidos en los pulmones observándose un aumento en la relación pulmón/peso corporal en 49,9\%, una disminución del residuo seco del $18,5 \%$ y una elevación del líquido pulmonar en $3,4 \%$. Estos datos revelan que existe un incremento de contenido de fluidos pulmonares que probablemente se deba a un mayor suministro sanguíneo y a la perfusión del propio tejido pulmonar ${ }^{(57,58)}$.

Asimismo, las cualidades tensoactivas disminuyen, el contenido total de los fosfolípidos se elevan, sobre todo la lisofosfatidilcolina y fosfatidiletanolamina, lo cual 
significa que existe un mecanismo de deterioro en las propiedades del surfactante debido al efecto detergente que tienen los lisofosfátidos en las membranas biológicas ${ }^{(57,58)}$. Bajo condiciones de microgravedad real en ratones que regresaron de un vuelo espacial de 30 días en el BION-M1biosatélite se observó que no hubo cambios significativos en las características del agente tensoactivo en las 12 horas posteriores del aterrizaje. Esto es, que los niveles de fosfolípidos y fosfatidilcolina se mantuvieron estables, pero el contenido del líquido pulmonar total aumentó ${ }^{(58)}$.

Por otro lado, las partículas procedentes de alimentos, telas, células de la piel y escape de aerosoles dentro de la nave espacial pueden ser aspiradas por los astronautas causando congestión en las vías respiratorias. Es por ello, que los sistemas de control ambiental de la atmosfera artificial dentro de la nave deben ser óptimos (presión, temperatura, humedad, ventilación y composición atmosférica) para garantizar las demandas de consumo metabólico de $\mathrm{O}_{2}$ y eliminar el $\mathrm{CO}_{2}$ fuera del vehículo a través de la "purga atmosférica". Además, la integridad estructural de la nave debe ser perfectamente cerrada para evitar contaminación atmosférica de la cabina por sustancias desconocidas como el polvo del suelo Marcianoy Lunar, pues se ha demostrado que causan enfermedades pulmonares intersticiales por sustancias tóxicas. El uso de protectores respiratorios, los filtros de carbono y el filtro de aire particulado (HEPA) sobre todo este último tiene una eficacia $99.97 \%$ evitando así el ingreso de partículas menores de 0.3 micras de tamaño ${ }^{(24,59)}$.

\section{Sistema renal}

El riñón es uno de los órganos que juega un papel fundamental en el balance de los líquidos y electrolitos. En condiciones de microgravedad, el volumen se contrae a la región intratorácica, lo cual nos llevaría pensar que la percepción de la sobrecarga de volumen por los barorreceptores ocasionaría disminución en la activación del "sistema renina-angiotensinavasopresina" generando diuresis y excreción de sodio. Sin embargo, ocurre lo contrario debido a la secreción de la hormona antidiurética en el hipotálamo por aumento en la osmolaridad sanguínea, reducción del volumen plasmático en un $17 \%$ y un estado de vasodilatación sistémica con consecuentes cambios fluctuantes de permeabilidad vascular. En general, los mecanismos de regulación hídrica en los astronautas son variables debido a cambios constantes de posición, hidratación y variabilidad de volumen sanguíneo por disminución de gravedad ${ }^{(22,24,60)}$.

La retención aguda de orina, infecciones de vías urinarias y principalmente los cálculos renales (litiasis) están incluidos en el $10 \%$ de todas las afecciones genitourinarias producto de estasis urinaria, deshidratación prolongada, dieta pobre en citratos $^{(61)}$ y por el remodelado óseo que genera un balance negativo de calcio ocasionándose precipitación de fracciones de oxalato de calcio $\left(\mathrm{CaC}_{2} \mathrm{O}_{4}\right)$ entonces surge así la posibilidad de que la evolución clínica sea desfavorable proporcionalmente al tamaño del lito durante y después del vuelo espacial ${ }^{(62,63)}$.

La alternativa efectiva para evitar la formación de cálculos renales, aparte del citrato de potasio y el pirofosfato añadido a la dieta del astronauta, es una adecuada y constante hidratación que mantenga el nivel urinario entre 2,5 a 3 litros/día ${ }^{(64)}$. En consecuencia, para minimizar el riesgo de enfermedad renal en el espacio se requiere desarrollar nuevas técnicas no invasivas, más seguras y rentables para la prevención, diagnóstico, control y tratamiento ${ }^{(22,65,66)}$.

\section{Sistema hematológico}

La exposición a SEP, GCR y a uno o más SPE, influyen directamente en la hematopoyesis, es decir, impiden el desarrollo normal de las células progenitoras de la sangre e inducen la génesis de leucemia ${ }^{(67)}$, incluso al desarrollo de enfermedades trombóticas ${ }^{(68)}$. En fases iniciales de exposición a la microgravedad y después de una rápida redistribución de líquidos se produce una disminución en el volumen plasmático y un aumento del hematocrito, esta rápida concentración de eritrocitos inhibe a la eritropoyetina y se reduce la masa de los glóbulos rojos, este proceso se denomina hemólisis selectiva lo que se conoce como anemia espacial| ${ }^{(69,70)}$, que ocurre posiblemente por una reducción del factor inducible por hipoxia (HIF-1a) y la expresión de Bcl-2 que es una proteína antiapoptótica alterada en condiciones ambientales espaciales. Este mismo fenómeno también ocurre en personas que residen en grandes alturas y que posteriormente descienden de forma rápida al nivel del mar, obteniéndose una reducción en el número de glóbulos rojos jóvenes ${ }^{(71)}$.

Las contramedidas para prevenir las afecciones hematológicas son la protección contra la radiación espacial, administración de eritropoyetina recombinante humana (EPO) para combatir la anemia espacial (relación neocito-macrófago) ${ }^{(72)}$ y el diseño e implementación de "los respirocitos" (glóbulos rojos artificiales) capaces de complementar o sustituir las funciones de los glóbulos rojos naturales ${ }^{(73)}$.

\section{Sistema inmunológico}

Se vio que los riesgos clínicos aumentan proporcionalmente a la permanencia en el espacio por una desregulación del sistema inmune producido por la exposición a la radiación galáctica, a partículas 
solares (rayos $\gamma$, rayos $X$ ) y al estrés inducido por la microgravedad $^{(74)}$, ocasionando alteraciones en la inmunidad celular, en la formación de leucocitos, citocinas e inhibición de la actividad fagocítica de granulocitos, monocitos y macrófagos, incluidas las subpoblaciones de linfocitos $\mathrm{T}$ ( $\mathrm{T}$ hellper 1, $\mathrm{T}$ hellper 2); B y NK. De igual forma, el factor nuclear kappa de células $B$ activadas (NF-Kb) se ven afectadas y disminuyen la producción de citocinas (IL-1, IL-6, IL-12 y Factor de necrosis tumoral alfa TNF-a $)^{(74,75,76)}$.

De manera similar, la microgravedad simulada deteriora la polarización de los tipos M1 y M2 a pesar de la estimulación con interferón gamma (INF- $\gamma$ ) e interleucina IL-4, ello conlleva a la inhibición de funciones de ambos tipos de macrófagos ${ }^{(77)}$.

Por consiguiente, los astronautas tienen más riesgo de infecciones por especies bacterianas (Escherichia, Bacillus, Enterobacter, Staphylococcus y Streptococcus)y hongos (Cándida, Aspergillus y Fusarium) ambos suficientemente aptos de modificar sus características de virulencia, reproducción y redistribución a zonas no habituales del cuerpo. Los virus de doble cadena también son capaces de reactivarse como: el Herpes Virus Simple tipo 1(HVS1), tipo 2 (HVS-2), Citomegalovirus (CMV), Virus de Epstein-Barr (VEB) y Virus de la Varicela Zóster (VVZ). Asimismo, tras un desequilibrio inmune se observó que algunos tripulantes experimentaron reacciones de hipersensibilidad persistentes además de erupciones cutáneas $^{(22,78,79)}$.

Por ello es crucial comprender la biología de la modulación inmune a fin de mantener la homeostasis bajo tales desafíos ${ }^{(80)}$. Por lo cual, para evitar la disfunción inmune es necesario la administración de vacunas prevuelo, mantener la ingesta de nutrientes optimizados, considerar inmunonutrición, antioxidantes, flavonoides, vitaminas $(A, C y E)^{(81,83)}$; factor estimulador de colonias de granulocitos (GCSF) (filgastrin, pegfilgastrin), antibióticos utilizados en vuelos espaciales (eritromicina, cefalexina, penicilina y tetraciclina) y otros medicamentos como antivirales ${ }^{(22,82,83)}$.

El desarrollo de la nanotecnología tiene un futuro prometedor en la exploración espacial, actualmente se propone como medida innovadora los nanotubos de carbono por su capacidad inmunoestimuladora y protección contra la radiación espacial. Por otra parte, el diseño del nanorobot médico o macrófago artificial "microbívoro", será útil para combatir infecciones, detección, reparación de células cancerosas y monitoreo celular. Ambas innovaciones serán benéficas para vuelos espaciales sobre todo de larga duración ${ }^{(84,85,86,87)}$.

\section{Sistema digestivo}

Los síntomas digestivos como náuseas y vómitos son característicos del vuelo espacial, pero el dato más curioso es la imposibilidad de eructar debido a que los gases no se pueden separar de los líquidos en el estómago en condiciones de microgravedad ${ }^{(88)}$.

La protección gástrica (mucopolisacáridos) disminuye con el aumento de la actividad de las células principales y parietales, en otras palabras, se incrementa la secreción de pepsinógeno; y del ácido clorhídrico $(\mathrm{HCl})$ cuyo potencial ulcerogénico en ambientes espaciales aún no está determinado ${ }^{(88)}$.

Igualmente existen alteraciones en la motilidad, microbiota intestinal, metabolismo de glucosa y lípidos ${ }^{(89,90)}$, daño de las vellosidades intestinales e interrupción en las uniones celulares epiteliales por la expresión de la proteína proapoptótica Bax y disminución de $\mathrm{BCl} 2$; alterando de esta manera su función defensiva, homeostasis y el riesgo de contraer enfermedades inflamatorias intestinales ${ }^{(76,91,92,93)}$ por el aumento de citosinas y disminución de la inmunoglobulina secretora $(\lg A)^{(76)}$.

Los probióticos son preferentes para mejorar el equilibro microbiano intestinal por su capacidad inductora en la conservación del sistema inmune ${ }^{(94,95)}$. Además, la gravedad artificial, ejercicios físicos de rutina e ingesta normal de alimentos cumplen un papel fundamental en el mantenimiento de la motilidad y equilibrio gastrointestinal( ${ }^{(98)}$.

En general, la farmacología en el campo de la medicina espacial es fundamental como contramedida, lo cual implica la optimización de medicamentos que se desempeñen de manera eficaz bajo la influencia de microgravedad y radiación sin afectar su biodisponibilidad ${ }^{(96)}$. Asimismo, la nutrición es imprescindible para garantizar el correcto funcionamiento de otros sistemas como: musculo-esquelético, hematológico, inmunológico, cardiovascular y neurosensorial e incluso mejorar la estabilidad psicosocial. Del mismo modo conserva el equilibrio en el gasto energético del astronauta que le permite mantenerse en condiciones de salud adecuadas

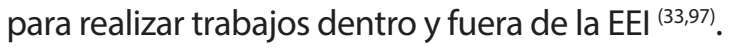

Los avances de la telemedicina y la medicina satelital sirven para brindar asistencia y monitorear los cambios fisiológicos de los diferentes sistemas del cuerpo que repercuten en la salud de los astronautas. Por lo tanto, es necesario el desarrollo de equipos biomédicos capaces de interactuar a grandes distancias del Planeta Tierra sobre todo en viajes espaciales de larga duración ${ }^{(99)}$. 
Tabla 1. Características de artículos originales incluidos.

\begin{tabular}{|c|c|c|c|}
\hline $\begin{array}{l}\text { Autor, año, número de } \\
\text { referencia }\end{array}$ & Población & Variables & Resultado \\
\hline Zhong, Guohui; 2016. ${ }^{(26)}$ & $\begin{array}{l}\text { Los experimentos se } \\
\text { llevaron a cabo en } \\
\text { ratones. } \\
\text { Modelo animal. }\end{array}$ & $\begin{array}{l}\text { Efectos de la microgravedad } \\
\text { simulada y remodelación } \\
\text { inducida en ventrículos } \\
\text { derecho e izquierdo. }\end{array}$ & $\begin{array}{l}\text { La microgravedad simulada induce la } \\
\text { remodelación cardiovascular a través de vías } \\
\text { de señalización intracelular como: HDAC4, } \\
\text { ERK } 1 / 2 \text {, LC3-II y AMPK estuvieron involucradas } \\
\text { en el proceso. }\end{array}$ \\
\hline
\end{tabular}

$\begin{array}{lll}\text { Palacios, Saúl; et al. 2019.(29) } & \begin{array}{l}\text { Registros de ECG } \\ \text { de 42 hombres } \\ \text { voluntarios. }\end{array} & \begin{array}{l}\text { La exposición a la } \\ \text { microgravedad a largo } \\ \text { plazo aumenta la } \\ \text { inestabilidad de la re- } \\ \text { polarización en la ECG. }\end{array}\end{array}$

Tejido muscular de 6

Radugina, E. A.; et al. 2018. ${ }^{(48)}$ ratones.

Modelo animal.

La exposición a microgravedad ocasiona atrofia y deterioro en la regeneración muscular.

Estudios celulares in vitro de astronautas de la NASA. (30-55 años).

Los experimentos se llevaron a cabo en ratones. Modelo animal.

Cultivos celulares in Shi, Lu; 2020.(77) vitro Modelo animal.

Registro de Imágenes de resonancia magnética archivados por la NASA.

Registros médicos archivados por la

Law, Jennifer; et al. 2016. ${ }^{(63)}$ NASA (Mercury, Gemini, Mir, Shuttle y EEI).

Los experimentos se Jin, Mingliang; et al. 2018. ${ }^{\text {(76) }}$ llevaron a cabo en ratas. Modelo animal.
Efectos directos de la radiación cósmica, solar y galáctica simulada repercute en las células madres hematopoyéticas.
La exposición a microgravedad prolongada muestra aumento significativo en la dinámica de repolarización periódica (PRD) asociado a riesgo de arritmias. Una contramedida basada en el entrenamiento físico contrarresta parcialmente estos cambios.

Los vuelos espaciales a largo plazo en microgravedad causaronatrofiay degeneración significativas del grupo muscular cuádriceps femoral, y pueden interferir con los procesos de regeneración muscular al inducir apoptosis en miofibrillas recién formadas durante su fase de diferenciación.

La exposición secuencial a protones e iones demostró ser mucho más perjudicial para la integridad de las células madre / progenitoras hematopoyéticas CD34 +. La radiación espacial afecta la hematopoyesis, induce la génesis de leucemia y reduce la formación de colonias in vitro.

Efectos de la microgravedad real y simulada en las propiedades, composición del factor surfactante y en el balance hídrico pulmonar.

Efectos de la microgravedad real y simulada en la diferenciación, calidad y polaridad funcional de macrófagos así como de las vías RAS/ERK/NFkB y P53.

La microgravedad real y simulada influye significativamente en las propiedades $y$ composición del factor surfactante, así como promueven el cambio en el equilibrio hídrico pulmonar.

La Microgravedad real y simulada inhiben significativamente la diferenciación de macrófagos de Células Progenitoras Hematopoyéticas (HPCs) y a la vez perjudican la polarización funcional de los macrófagos (M1/M2), activa la vía de P53 y regula la vía RAS/ERK/NFkB

Cambios de estructura y función del cerebro humano en condiciones de microgravedad.

Los cambios estructurales del cerebro humano por influencia del ambiente espacial están asociados con el puntaje de pruebas cognitivas y motoras.

Los astronautas de la NASA han tenido el

Condiciones urinarias de los astronautas asociados a vuelos espaciales.

Respuesta de la barrera epitelial intestinal en microgravedad simulada. mayor número de casos por cálculos renales después del vuelo, el cociente de probabilidad femenino y masculino es $11 / 14$. Un astronauta con retención de orina tiene un riesgo de 25 veces más para desarrollar infección urinaria.

La microgravedad simulada indujo a la susceptibilidad e interrupción de la barrera epitelial a nivel intestinal, destrucción de las vellosidades intestinales a través de la apoptosis por la sobreexpresión de Bax, y disminución de la proteína anti apoptótica Bcl2. 


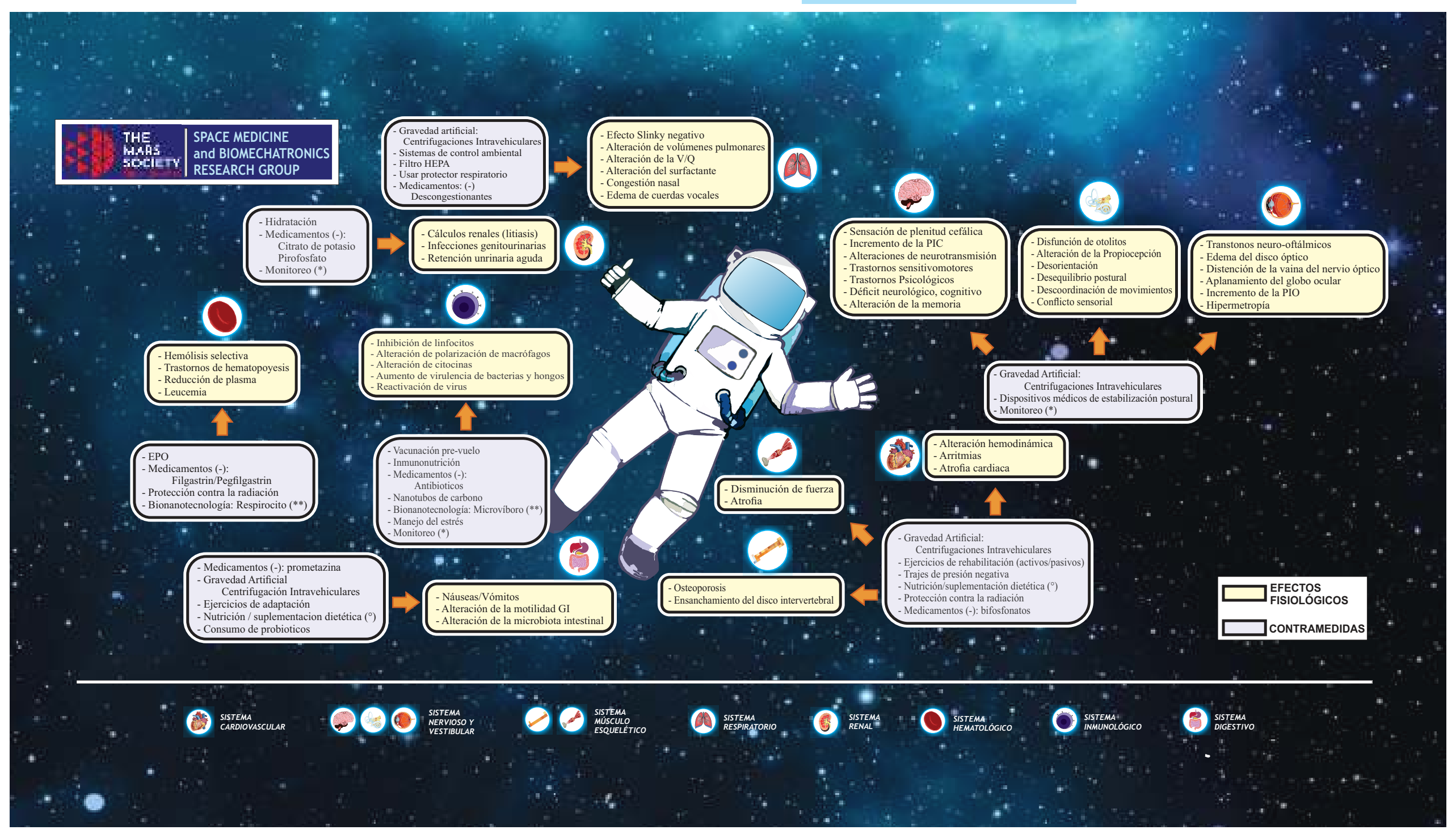

(-) Medicamentos necesarios para kit biomédico en viajes espaciales (analgésicos, antiinflamatorios, antihistamínicos, antibióticos, antivirales) específicos para cada sistema. (') Nutriente (proteínas, carbohidratos y lípidos). Suplementos: vitaminas, antioxidantes, flavonoides, ácidos grasos, minerales.

(*) Monitoreo a través de la telemedicina: Evaluación clínica, laboratorio (perfil inmunológico, serológico y hematológico), psicológico y diagnóstico por imagen.

(**) Bionanotecnología en desarrollo.

Figura 1. Astronauta con múltiples efectos fisiológicos en el espacio y sus contramedidas respectivas. 


\section{CONCLUSIÓN}

Los cambios fisiológicos en el espacio, son desafíos que la Medicina Espacial debe enfrentar formulando nuevas hipótesis en relación a las contramedidas como alternativas de prevención y tratamiento en la atención sanitaria de los astronautas.

Los hallazgos principales de la presente revisión evidencian cómo la microgravedad y la exposición permanente a la radiación, podrían llegar a ser letales en el espacio ${ }^{(8)}$, donde los efectos en la fisiología humana son considerables (Figura 1) y ocasionarían múltiples problemas estructurales y genéticos. Los cuales se presentan en dos fases: temprana y tardía.

En la fase temprana, los efectos más notorios se muestran a nivel del sistema cardiovascular donde se presenta una redistribución de líquidos por microgravedad, generando problemas en la hemodinamia que repercuten en el sistema nervioso produciendo síntomas neurológicos por cambios en la neurotransmisión y aumento de la PIC, los mismos que conllevan a trastornos sensitivomotores, neurovestibulares y visuales. También, el aumento de volumen en la región cefálica provoca congestión nasal, edema facial y de cuerdas vocales ${ }^{(21,44)}$. De hecho los mejores métodos para prevenir y rehabilitar dichas afecciones son los ejercicios pasivos/activos, trajes de presión negativa en los miembros inferiores y gravedad artificial a través de centrifugaciones intravehiculares ${ }^{(30,31,44)}$.

En la fase tardía, dentro del sistema cardiovascular se observa atrofia cardiaca. A nivel del sistema musculoesquelético ocurre la reabsorción ósea y deterioro muscular, los mismos que pueden evitarse con los métodos mencionados en el párrafo anterior y ejercicios de resistencia. Adicionalmente, se afirman que los bifosfonatos y la nutrición con alto contenido proteico son de suma importancia para conservar el gasto energético del astronauta; igualmente, contribuyen a mejorar la anatomía y fisiología osteomuscular ${ }^{(54,55)}$.

A nivel del sistema renal, la litiasis se incluye en el 10\% de las afecciones genitourinarias, esta es producida por la reabsorción de calcio, hipercalciuria, deshidratación e hipocitraturia ${ }^{(61,62)}$. En contramedida, se describe que la hidratación y la dieta alta en citrato de potasio es efectiva para evitarla ${ }^{(64)}$.

En el sistema pulmonar se producen alteraciones en los volúmenes / capacidades pulmonares y su vascularización sufre cambios adaptativos anatómicos más que fisiológicos ${ }^{(56)}$.

Autores mencionan que la exposición a microgravedad yespecialmentelaradiación repercutennegativamente en la hematopoyesis y linfopoyesis ${ }^{(67,74)}$, esta última alteración está asociada a infecciones por parte del microbiota existente en la piel, mucosa e intestinos ${ }^{(76)}$. En consecuencia, aumenta la proliferación bacteriana, micótica e incluso los virus como el VHS, VEB y VVZ se reactivan ${ }^{(78)}$. Para ello científicos plantean que la vacunación pre-vuelo, medicamentos e inmunonutrición mejoran la respuesta inmune $\mathrm{e}^{(78,81,83,86)}$. Asimismo, diseñar materiales a escala nanométrica (bio-nanotecnología) como respirocitos y microbívoros (actualmente en desarrollo), serían efectivos para su aplicación en patologías de los sistemas hematológico e inmunológico respectivamente ${ }^{(73,84,87)}$.

En síntesis, se alienta a investigar y desarrollar nuevas contramedidas efectivas para estos efectos fisiológicos con el objetivo de seguir explorando el espacio, como los viajes a la Luna y Marte. Por ello, son necesarias nuevas tecnologías biomédicas, procedimientos y protocolos clínicos para prevenir, diagnosticar, tratar y salvaguardar el bienestar del astronauta ante los posibles riesgos del ambiente espacial y su repercusión en el cuerpo humano. De igual forma, los avances en el estudio de la fisiología durante viajes espaciales contribuyen a descubrir mejores tratamientos para preservar la salud de la población en nuestro Planeta Tierra.

Contribuciones de autoría: Los autores participaron en la génesis de la idea, diseño de proyecto, recolección e interpretación de datos, análisis de resultados y preparación del manuscrito del presente trabajo de investigación.

\section{Financiamiento: Autofinanciado.}

Conflicto de interés: Los autores declaran no tener conflicto de interés en la publicación de este artículo.

Recibido: 05 de febrero 2020

Aprobado: 25 de marzo 2020

Correspondencia: Milton V. Rivera.

Dirección: Jr. Revolución, Mariano Melgar, San Miguel, San Román, Puno-Perú. Teléfono: +51931983737

Correo: vizariveramilton@gmail.com 


\section{REFERENCIAS BIBLIOGRÁFICAS}

1. Carrillo-Esper R. Medicina Espacial. Cirugía y Cirujanos. 2014 Octubre 10; 82(591593): p. 591-593.

2. López Negro E. La casa del Espacio: habitar fuera de la Tierra. E.T.S. Arquitectura (UPM). 2017 Agosto 28: p. 1-111.

3. Carrillo-Esper R. Reanimación cardiopulmonar en microgravedad. Revista Mexicana de Anestesiología. 2016 Abril-Junio 1; 39: p. 8-10.

4. Pérez Sastre J, Rodrigo Villa J. Medicina Espacial. 2018 Octubre 21: p. 1-17.

5. Stepanek J, Blue RS, Parazynski S. Space Medicine in the Era of Civilian Spaceflight. The New England Journal of Medicine. 2019 March; 380: p. 1053-1060.

6. Dhwani V J, Raosaheb K K, Rana P. S. Microgravity alters cancer growth and progression. Current Cancer Drug Targets. 2014 January; 14(4): p. 394-406.

7. Muñiz LM. Cooperación internacional. Cuadernos de estrategia. 2014;(170): p. 93 118.

8. Yatagai F, Honma M, Ishioka N, Dohmae N. Combined effects of radiation and microgravity. Hoshasen Seibutsu Kenkyu (Online). 2017; 52(1): p. 80-94.

9. Charles W L, K K, Saffold Fitzpatrick M, Mukvaney J. Space Radiation. [Online] [cited 20190118 [NASA Human Research Program Engagement]. Available from HYPERLINK "https://www.nasa.gov/sites/default/files/atoms/files/space_radiation ebook.pdf".

10. Di Fino L, Zaconte V, Stangalini M, Sparvoli R, Picozza P, Piazzesi R, et al. Solar particle event detected by ALTEA on board the International Space Station. Journa of Space Weather and Space Climate. 2014 May; 4: p. A19.

11. Dorling K. The Stars - The Definitive Visual Guide To The Cosmos. 1st ed. Page M Meeus C, setford S, O'Hara S, editors. Londres: Penguin Random House; 2017.

12. MSFC JW. What Is Microgravity? [Online].; 2015-06-16T10:33-04:00 [cited 2020 01 18. Available from: HYPERLINK:http://www.nasa.gov/audience/forstudents/5-8/ features/nasa-knows/what-is-microgravity-58.html

13. Carrillo Esper R, Díaz Ponce Medrano JA, Peña Pérez CA, Flores Rivera O Ortiz Trujillo A, Cortés Antonio $O$, et al. Efectos fisiológicos en un ambiente de microgravedad. Revista de la Facultad de Medicina (México). 2015; 58(3): p. 13-24.

14. Ramírez Jiménez SI. Astrobiología y medicina espacial. In Intersistemas SA editor. Medicina Espacial. México: Conacyt; 2014-2016. p. 87-98.

15. Hodkinson PD, Anderton RA, Posselt BN, Fong KJ. An overview of space medicine. BJA: British Journal of Anaesthesia. 2017 December; 119: p. i143-i153.

16. Karrim N, Magula N, Saman Y. Antihistamines for motion sickness. The Cochrane Database of Systematic Reviews. 2017 July 07; 2017(7).

17. Ortega HJ, Harm DL, Reschke MF. Space and Entry Motion Sickness. In Barrat MR, Baker ES, Pool SL, editors. Principles of Clinical Medicine for Space Flight. New York, NY: Springer; 2019. p. 441-456.

18. Reschke MF, Cohen HS, Cerisano JM, Clayton JA, Cromwell R, Danielson RW, et al. Effects of Sex and Gender on Adaptation to Space: Neurosensory Systems. Journa of Women's Health. 2014 April 01; 23(11): p. 959-962.

19. Kornilova LN, Naumov IA, Glukhikh DO, Ekimovskiy GA, Pavlova AS, Khabarova VV, et al. Vestibular function and space motion sickness. Human Physiology. 2017 October; 43(5): p. 557-568.

20. Ling S, Li Y, Zhong G, Zheng Y, Xu Q, Zhao D, et al. Myocardial CKIP-1 Overexpression Protects from Simulated Microgravity-Induced Cardiac Remodeling. Frontiers in Physiology. 2018 January 25; 9.

21. Myers J, Wermer C, Nelson E, Feola a, Raykin J, Samuels B, et al. Modeling Microgravity Induced Fluid Redistribution Autoregulatory and Hydrostatic Enhancements. ntrs.nasa.gov. 2017 January 23.

22. Martin J, Nikolett M. Urgencias Medicas en los vuelos espaciales. In Intersistemas SA, editor. Medicina Espacial. Mexico: Conacyt; 2014-2016. p. 291-297.

23. Meza Márquez JM, Carrillo Esper R. Sistema cardiovascular. In Intersistemas SA editor. Medicina Espacial. México: Conacyt; 2014-2016. p. 139-164

24. Baker ES, Barratt MR, Sams CF, Wear ML. Human Response to Space Flight. In Barratt MR, Baker ES, Pool SL, editors. Principles of Clinical Medicine for Space Flight. New York, NY: Springer; 2019. p. 367-411.

25. Iglesias Leal R. Perfil del hombre cósmico. In Intersistemas SA, editor. Medicin Espacial. México: Conacyt; 2014-2016. p. 73-85.

26. Zhong G, Li Y, Li H, Sun W, Cao D, Li J, et al. Simulated Microgravity and RecoveryInduced Remodeling of the Left and Right Ventricle. Frontiers in Physiology. 2016 June $29 ; 7$

27. Delp MD, Charvat JM, Limoli CL, Globus RK, Ghosh P. Apollo Lunar Astronauts Show Higher Cardiovascular Disease Mortality: Possible Deep Space Radiation Effects on the Vascular Endothelium. Scientific Reports. 2016; 6(1): p. 1-11.

28. Hatzistergos E, Jiang Z, Valasaki K, Takeuchi LM, Balkan W, Atluri P, et al. Simulated Microgravity Impairs Cardiac Autonomic Neurogenesis from Neural Crest Cells. Stem Cells and Development. 2018 January 16; 27: p. 819-830.
29. Palacios S, Caiani EG, Landreani F, Martínez JP, Pueyo E. Long-Term Microgravity Exposure Increases ECG Repolarization Instability Manifested by Low-Frequency Oscillations of T-Wave Vector. Frontiers in Physiology. 2019 December; 10: p. 1510.

30. Gunga $H C$, Ahlefeld VWv, Appell Coriolano HJ, Werner A, Hoffmann U. Countermeasures. In Gunga HC, Ahlefeld VW, Appell Coriolano HJ, Werner A Hoffmann U, editors. Cardiovascular System, Red Blood Cells, and Oxygen Transport in Microgravity.: Springer International Publishing; 2016. p. 67-74.

31. Norsk P, Shelhamer M. Bringing Gravity to Space. NASA technical Reports Server (NTRS). 2016 April 24.

32. Quiñenao CD. El ejercicio físico,una contramedida en condiciones de microgravedad. instname:Universidad FASTA. 2015 Mayo.

33. Hughson RL, Helm A, Durante M. Heart in space: effect of the extraterrestrial environment on the cardiovascular system. Nature Reviews Cardiology. 2018 March; 15(3): p. 167-180.

34. Nday CM, Frantzidis C, Jackson G, Bamidis P, Kourtidou-Papadeli C. Neurophysiological changes in simulated microgravity: An animal model. Neurology India. 2019 May; 67(8): p. 221.

35. Van Ombergen, Demertz , Tomilovskaya, Jeurissen, Sijbers, Kozlovskaya IB, et al. The effect of spaceflight and microgravity on the human brain. Journal of Neurology. 2017 October 01; 256(1): p. 18-22.

36. Pérez Calatayud ÁA. Sistema neurologico y vestibular. In Bravo Valdez A, editor. Medicina Espacial. México: Conacyt; 2014-2016. p. 153-164.

37. Roberts D, Asemani D, Nietert PJ, Eckert MA, Inglesby DC, Bloomberg JJ, et al. Prolonged Microgravity Affects Human Brain Structure and Function. American Journal of Neuroradiology. 2019 November; 40(11): p. 1878-1885.

38. Wang T, Chen H, Lv K, Ji G, Zhang Y, Wang Y, et al. iTRAQ-based proteomics analysis of hippocampus in spatial memory deficiency rats induced by simulated microgravity. Journal of Proteomics. 2017 May 8; 160: p. 64-73.

39. Vega R, Soto E, Pliego A, Alexandrov V, Alexandrova T. Dispositivos para la estabilización de la postura en microgravedad. In Intersistemas SA, editor. Medicina Espacial. México: Conacyt; 2014-2016. p. 121-137.

40. Reschke MF, Clément G. Vestibular and Sensorimotor Dysfunction During Space Flight. Current Pathobiology Reports. 2018 September 01; 6(3): p. 177-183.

41. Marshall-Goebel K, Damani R, Bershad EM. Brain Physiological Response and Adaptation During Spaceflight. Neurosurgery. 2019; 0(1): p. 1-7.

42. Lee AG, Mader TH, Gibson CR, Tarver W. Space Flight-Associated Neuro-ocula Syndrome. JAMA Ophthalmology. 2017 July 20; 135(9): p. 992-994.

43. Kreutzberg G, Rosenberg M, Peters B, Reschke M. Validation of a Manually Oscillating Chair for In-The-Field Assessment of Dynamic Visual Acuity on Crewmembers Within Hours of Returning From Long-Duration Spaceflight. NASA Technical Reports Server (NTRS). 2017 January 23.

44. Zhang LF, Hargens AR. Spaceflight-Induced Intracranial Hypertension and Visual Impairment: Pathophysiology and Countermeasures. Physiological Reviews. 2017 November 22; 98(1): p. 59-87.

45. Markolefa I, Lambrou GI. The role of autophagy during osteoclastogenesis under microgravity conditions. International Journal of Astrobiology. 2018; 18(4): p. 384-390.

46. Gambara G, Salanova M, Ciciliot S, Furlan S, Gutsmann M, Schiffl G, et al. Gene Expression Profiling in Slow-Type Calf Soleus Muscle of 30 Days Space-Flown Mice. PLOS ONE. 2017 January 11; 22(1).

47. Harding C, Takemoto J, Vargis E. In Vitro Modeling of Microgravity-Induced Muscle Atrophy and Spaceflight Radiation. Utah NASA Space Grant Consortium. 2016 May;: p. 1-11

48. Radugina EA, Almeida EaC, Blaber E, Poplinskaya VA, Markitantova YV, Grigoryan EN. Exposure to microgravity for 30 days onboard Bion M1 caused muscle atrophy and impaired regeneration in murine femoral Quadriceps. Life Sciences in Space Research. 2018 February; 16: p. 18-25

49. Beheshti A, Ray S, Fogle HW, Berrios DC, Costes SV. Systemic Microgravity Response: Utilizing GeneLab to Develop Hypotheses for Spaceflight Risks. NASA Technical Reports Server (NTRS). 2017 October 25.

50. Sambandam Y, Townsend MT, Pierce JJ, Lipman CM, Haque A, Bateman TA, et al. Microgravity control of autophagy modulates osteoclastogenesis. Bone. 2014 April; 61: p. 125-131.

51. Demontis GC, Germani MM, Caiani EG, Barravecchia I, Passino C, Angeloni D. Human Pathophysiological Adaptations to the Space Environment. Frontiers in Physiology. 2017 August 02; 8(547).

52. Nordberg RC, Bodle JC, Loboa G. Mechanical Stimulation of Adipose-Derived Stem Cells for Functional Tissue Engineering of the Musculoskeletal System via Cyclic Hydrostatic Pressure, Simulated Microgravity, and Cyclic Tensile Strain. In SpringerLink, editor. Adipose-Derived Stem Cells. New York, NY: Springer New York; 2018. p. $215-230$

53. Luan H, Huang Y, Li J, Sun L, Fan Y. Effect of Local Vibration and Passive Exercise on the Hormones and Neurotransmitters of Hypothalamic-Pituitary-Adrenal Axis in Hindlimb Unloading Rats. Microgravity Science and Technology. 2018 August 12; 30(4): p. 483-489. 
54. Grimm D, Grosse J, Wehland M, Mann V, Reseland JE, Sundaresan A, et al. The impact of microgravity on bone in humans. Bone. 2016 June; 87: p. 44-56.

55. Tanaka K, Nishimura N, Kawai Y. Adaptation to microgravity, deconditioning, and countermeasures. The Journal of Physiological Sciences. 2017 March 01; 67(2): p. 271-281.

56. Prisk GK. Microgravity and the respiratory system. The European Respiratory Journal. 2014 May; 43(5): p. 1459-1471.

57. Bryndina IG, Vasilieva NN, Krivonogova YA, Baranov VM. Effect of Long-Term Simulated Weightlessness on Surfactant and Water Balance in Mouse Lungs. Bulletin of Experimental Biology and Medicine. 2013 July 01; 155(3): p. 306-308.

58. Kazarin D, Bryndina I, Vasilieva N. The properties of pulmonary surfactant afte 30-day space flight and simulated microgravity. European Respiratory Journal. 2015; 46(suppl 59).

59. Beck G, Law J, Bacal K, Barratt MR. Hypoxia, Hypercarbia, and Atmospheric Control. In Barratt MR, Baker ES, Pool SL, editors. Principles of Clinical Medicine fo Space Flight. New York, NY: Springer; 2019. p. 109-151.

60. Diaz de León Ponce MA, Moreno Santillán AA, Brioñes Garduño JC. Líquidos, electrolitos y función renal en el Espacio. In Intersistemas SA, editor. Medicina Espacial. México: Conacyt; 2014-2016. p. 185-172.

1. Carrillo-Esper R, Carrillo-Cordova LD, Villena-López EL, Carrillo-Cordova DM. Urología en el espacio y cambios genitourinarios en el ambiente de microgravedad. Rev Mex Urol. 2018 Marzo-Abril; 87(2): p. 162-167.

62. Kassemi M, Griffin E, Thompson D. Numerical assessment of $\mathrm{CaOx}$ renal calculi development in space using PBE coupled to urinary flow and species transport. International Journal of Heat and Mass Transfer. 2018 June; 121: p. 1146-1158.

63. Law J, Cole R, Young MH, Mason S. NASA Astronaut Urinary Condition Associated with Spaceflight. NASA Technical Reports Server (NTRS). 2016 April 24.

64. Kassemi M, Thompson D. Prediction of renal crystalline size distributions in space using a PBE analytic model. 2. Effect of dietary countermeasures. American Journal of Physiology-Renal Physiology. 2016 June 8; 311(3): p. F531-F538.

65. Antonsen E, Pietrzyk R. Programmatic Considerations to Reduce the Risk of Adverse Renal Stone Events in Spaceflight. NASA Technical Reports Server (NTRS) 2017 May 12.

66. Chang HC Sorensen M Harper JD, Bailey MR, Donoviel D. Re: Leapman et al.: Up and Away: Five Decades of Urologic Investigation in Microgravity (Urology 106:1825). Urology. 2017 December; 110(265-266): p. 265-266

67. Rodman C, Almeida-Porada G, George SK, Moon J, Soker S, Pardee T, et al. I vitro and in vivo assessment of direct effects of simulated solar and galactic cosmic radiation on human hematopoietic stem/progenitor cells. Leukemia. 2017 November 24; 31(6): p. 1398-1407.

68. Romero-Weaver AL, Lin L, Carabe-Fernandez A, Kennedy AR. Effects of Sola Particle Event-Like Proton Radiation and/or Simulated Microgravity on Circulating Mouse Blood Cells. Gravitational and space research : publication of the American Society for Gravitational and Space Research. 2014 August; 2(1): p. 42-53.

69. Gunga HC, Ahlefeld VWv, Appell Coriolano HJ, Werner A, Hoffmann U. Red Blood Cells in Space. In Cardiovascular System, Red Blood Cells, and Oxygen Transport in Microgravity. Cham: Springer International Publishing; 2016. p. 35-55.

70. Rosales Gutiérrez AO, Espinosa de los Montesinos Estrada I. Cambios Hematológicos en microgravedad. In Intersistemas SA, editor. Medicina Espacial. México: Conacyt; 2014-2016. p. 345-350.

71. Mairbäurl H. Neocytolysis: How to Get Rid of the Extra Erythrocytes Formed by Stress Erythropoiesis Upon Descent From High Altitude. Frontiers in Physiology. 2018 April 05; 9(345).

72. Amir NA, Amir K, Mahdieh D, Akbar N, Abbas N. The effect of erythropoietin on rat's red blood cell indices in simulated microgravity (experimental study). 2016 January $01 ; 14(1):$ p. $22-26$

73. Arpita J, Hinali T, Stanukumar B, Krunali T, D.B M. Nanotechnology revolution:respirocytes and its application in life sciences. Innovare Journal of Life Science. 2013; 1(1): p. 8-13.

74. Daila S G, Asma R, Xian LO, Adeola Y M, George B C, Peter K, et al. Variable Hematopoietic Responses to Acute Photons, Protons and Simulated Sola Particle Event Protons. International Institute of Anticancer Research (Dr. John G. Delinassios), All rights reserved. 2007 December 31; 22(2).

75. Zhang $\mathrm{Y}$, Moreno Villanueva $M$, Krieger $S$, Ramesh GT, Neelam $S$, Wu H Trascriptómica, via NF-kB y sus posibles consecuencias para la salud relacionada con los vuelos espaciales. International Journal of Molecular Sciences. 2017 May 18(6): p. 1166.

76. Jin M, Zhang H, Zhao K, Xu C, Shao D, Huang Q, et al. Responses of Intestina Mucosal Barrier Functions of Rats to Simulated Weightlessness. Frontiers in Physiology. 2018 June 14; 9(729).
77. Shi L, Tian H, Wang P, Li L, Zhang Z, Zhang J, et al. Spaceflight and simulated microgravity suppresses macrophage development via altered RAS/ERK/NFKB and metabolic pathways. Cellular \& Molecular Immunology. 2020 January.

78. Crucian BE, Chouker A, Simpson J, Mehta S, Marshall G, Smith SM, et al. Immune System Dysregulation During Spaceflight: Potential Countermeasures for Deep Space Exploration Missions. Frontiers in Immunology. 2018 June 28; 9.

79. Sams CF, Crucian BE, Pierson DL. Immunology and Microbiology. In Barratt MR Baker ES, Pool SL, editors. Principles of Clinical Medicine for Space Flight. New York, NY: Springer; 2019. p. 659-671.

80. Frippiat JP, Crucian BE, Quervain DJFd, Grimm D, Montano N, Praun S, et al. Towards human exploration of space: The THESEUS review series on immunology research priorities. npj Microgravity. 2016 August; 2(1).

81. Makedonas G, Mehta S, Choukèr A, Simpson RJ, Marshall G, Orange JS, et al Specific Immunologic Countermeasure Protocol for Deep-Space Exploration Missions. Frontiers in Immunology. 2019 October 11; 10(2407).

82. Romero-Weaver AL, Lin L, Carabe-Fernandez A, Kennedy AR. Effects of Solar Particle Event-Like Proton Radiation and/or Simulated Microgravity on Circulating Mouse Blood Cells. Gravitational and space research : publication of the American Society for Gravitational and Space Research. 2014 August; 2(1): p. $42-53$.

83. Heer M, Baecker N, Smith SM, Zwart SR. Nutritional Countermeasures for Spaceflight-Related Stress. In Chouker A. Stress Challenges and Immunity in Space: From Mechanisms to Monitoring and Preventive Strategies. Berlín Heidelberg: Springer International Publishing; 2020. p. 593-616.

84. Crescio C, Orecchioni M, Ménard-Moyon C, Sgarrella F, Pippia P, Manetti R, et al. Immunomodulatory properties of carbon nanotubes are able to compensate immune function dysregulation caused by microgravity conditions. Nanoscale. 2014 July 24; 616: p. 9599-9603.

85. Meyyappan M, Koehne JE, Han JW. Nanoelectronics and nanosensors for space exploration. MRS Bulletin. 2015 October; 40(10): p. 822-828.

86. Kennedy AR. Biological effects of space radiation and development of effective countermeasures. Life Sciences in Space Research. 2014 April 1; 1: p. 10-43.

87. Mishra S. Nanotechnology in medicine. Indian Heart Journal. 2016 May-June 14 68(3): p. 437-439.

88. Pascal B. The Stomach - Both a target and potential contributor in space sickness. In Kucharczyk J, Stewart DJ, Miller AD, editors. Nausea and Vomiting: Recent Research and Clinical Advances.: CRC Press; 2017. p. 260

89. Shi J, Wang Y, He J, Li P, Jin R, Wang K, et al. Intestinal microbiota contributes to colonic epithelial changes in simulated microgravity mouse model. The FASEB Journal. 2017 June; 31(8): p. 3695-3709.

90. Wang $Y$, Zhao W, Shi J, Wang J, Hao J, Pang $X$, et al. Intestinal microbiota contributes to altered glucose metabolism in simulated microgravity mouse model. The FASEB Journal. 2019 june; 33(9): p. 10140-10151.

91. Wang S, Zhang Y, Guo J, Kang L, Deng Y, Li Y. Investigation on rat intestinal homeostasis alterations induced by 7-day simulated microgravity effect based on a proteomic approach. Acta Astronautica. 2020 January; 166: p. 560-566.

92. Li P, Shi J, Zhang P, Wang K, Li J, Liu H, et al. Simulated microgravity disrupts intestinal homeostasis and increases colitis susceptibility. The FASEB Journal. 2015 April; 29(8): p. 3263-3273.

93. Alvarez R, Stork CA, Sayoc-Becerra A, Marchelletta RR, Prisk GK, McCole DF. A Simulated Microgravity Environment Causes a Sustained Defect in Epithelial Barrier Function. Scientific Reports. 2019 November; 9(1): p. 1-15.

94. Stahl S, Voorhies A, Lorenzi H, Castro-Wallace S, Douglas G. Effect of Low Shea Modeled Microgravity (LSMMG) on the Probiotic Lactobacillus Acidophilus ATCC 4356. NASA Technical Reports Server (NTRS). 2016 February 08.

95. Sakai T, Moteki Y, Takahashi T, Shida K, Kiwaki M, Shimakawa Y, et al. Probiotics into outer space: feasibility assessments of encapsulated freeze-dried probiotics during 1 month's storage on the International Space Station. Scientific Reports. 2018 July; 8(1): p. 1-11

96. Putcha L, Taylor PW, Daniels VR, Pool SL. Clinical Pharmacology and Therapeutics. In Nicogossian AE, Williams RS, Huntoon CL, Doarn CR, Polk JD, Schneider VS, editors. Space Physiology and Medicine: From Evidence to Practice. New York, NY: Springer; 2016. p. 323-346.

97. Enrico C. Space nutrition: the key role of nutrition in human space flight arXiv:1610.00703 [q-bio]. 2016 October.

98. Laurens C, Vernikos J, Gauquelin-Koch G, Blanc S, Bergouignan A. Revisiting the Role of Exercise Countermeasure on the Regulation of Energy Balance During Space Flight. Frontiers in Physiology. 2019 March; 10.

99. Hernández Marroquín. Telemedicina y medicina satelital. In Intersistemas SA editor. Medicina espacial. México: Conacyt; 2014-2016. p. 55-71. 